\title{
Autoeficácia na formação superior: seu papel preditivo na satisfação com a experiência acadêmica
}

\author{
Self-efficacy on higher education: Its predictive \\ role to satisfaction with academic experience
}

\author{
Acácia Aparecida Angeli dos SANTOS ${ }^{1}$ iD) 0000-0002-8599-7465 \\ Cristian ZANON² ID 0000-0003-3822-5275 \\ Vanessa Domingues ILHA ${ }^{1}$ iD 0000-0001-7384-347X
}

\section{Resumo}

Este estudo tem como objetivo avaliar se a autoeficácia na formação superior pode predizer a satisfação com a experiência acadêmica. Participaram do estudo 372 universitários (66,4\% do sexo feminino) de uma instituição de ensino superior paulista, com idade entre 17 e 53 anos $(M=22,84 ; D P=6,09)$. Os instrumentos utilizados foram as Escalas de Autoeficácia na Formação Superior e a Escala de Satisfação com a Experiência Acadêmica. Uma análise de regressão múltipla indicou que o conjunto das facetas da autoeficácia acadêmica explicou grande parte da variância da satisfação acadêmica total (aproximadamente 64\%), sendo que a faceta interação social apresentou coeficiente de regressão elevado $(\beta=0,79$; $p<0,001)$. Esses resultados indicam que a autoeficácia é uma importante preditora da satisfação acadêmica.

Palavras-chave: Adaptação acadêmica; Autoeficácia; Bem-estar; Teoria social cognitiva; Universidades.

\begin{abstract}
This study aims to evaluate the possible predictive role of self-efficacy in higher education regarding student satisfaction with the academic experience. Three hundred and seventy-two university students from the state of São Paulo (66.4\% female) participated this study, aged between 17 - 53 years $(M=22.84, S D=6.09)$. The instruments used were the Higher Education Self-efficacy Scale and the Academic Experience Satisfaction Scale. A multiple regression analysis indicated the facets of academic self-efficacy explained considerable amount of variance in academic satisfaction (approximately

1 Universidade São Francisco, Departamento de Psicologia, Programa de Pós-Graduação em Psicologia. Campinas, SP, Brasil.

2 Universidade Federal do Rio Grande do Sul, Instituto de Psicologia, Programa de Pós-Graduação em Psicologia. R. Ramiro Barcelos, 2600, 90035-002, Porto Alegre, RS, Brasil. Correspondência para/Correspondence to: C. ZANON. E-mail: <crstn.zan@gmail.com>.

Como citar este artigo/How to cite this article

Santos, A. A. A., Zanon, C., \& Ilha, V. D. (2019). Autoeficácia na formação superior: seu papel preditivo na satisfação com a experiência acadêmica. Estudos de Psicologia (Campinas), 36, e160077. http://dx.doi.org/10.1590/1982-0275201936el60077
\end{abstract}


$64 \%)$, and the social interaction self-efficacy facet presented a high regression coefficient $(\beta=0.79 ; p<0.001)$. These findings indicate that self-efficacy constitutes an important predictor of academic satisfaction.

Keywords: Academic adaptation; Self-efficacy; Well-being; Social cognitive theory; Universities.

O sucesso e a satisfação acadêmica constituem construtos de interesse para profissionais que trabalham com o desenvolvimento de carreira. Universitários satisfeitos com as atividades acadêmicas e a escolha profissional constituiriam a situação ideal para a aquisição de conhecimentos e habilidades essenciais ao desenvolvimento da profissão futura (Ambiel, 2015; Bardagi \& Hutz, 2012; Magalhães \& Teixeira, 2013). Contudo, o ingresso no ensino superior pode estar associado a uma série de conflitos e crises. Muitos deixam de morar com os pais e necessitam rearranjar a rede de amizades. Ademais, as novas obrigações e demandas do meio acadêmico podem envolver habilidades de estudo e controle emocional ainda pouco desenvolvidas (Carlotto, Teixeira, \& Dias, 2015; Ourique \& Teixeira, 2012). Dessa forma, é essencial a investigação de fatores que possam contribuir para a adaptação e satisfação acadêmicas.

Uma boa escolha profissional perpassa a forma como o sujeito toma suas decisões e como lida com as consequências afetivas e cognitivas produzidas. Embora a formação profissional não seja pré-requisito para um futuro de sucesso, fatores vocacionais são importantes para a realização pessoal e podem auxiliar no processo de adaptação ao contexto universitário. Muitas pessoas optam por determinadas carreiras sem conhecê-las integralmente em termos de tarefas, responsabilidades e dificuldades (Bardagi \& Hutz, 2012; Teixeira, Castro, \& Zoltowski, 2012). É sabido que alguns fatores são relevantes para obtenção de uma formação superior bem-sucedida. Neste trabalho, a questão a ser investigada diz respeito ao quanto a autoeficácia na formação superior pode ser preditora da satisfação acadêmica em estudantes universitários.

\section{Autoeficácia}

A autoeficácia é um conceito de destaque na Teoria Social Cognitiva de Bandura (1977), porque trata da crença que as pessoas têm sobre si mesmas. Ela pertence à classe de expectativas que estão ligadas ao self e representa um importante fator na execução das tarefas e nas decisões que os sujeitos tomarão ao longo de suas vidas. Acredita-se que quanto maior a autoeficácia percebida, maior o grau de esforço investido e a persistência em alcançar uma meta estabelecida.

A definição de autoeficácia proposta por Bandura (1986, 1993, 1997) consiste no julgamento das próprias capacidades de executar ações exigidas para se atingir uma dada performance. Na área acadêmica, o conceito não difere muito, pois crenças de autoeficácia são convicções pessoais em dar conta de uma tarefa em um grau de qualidade definida (Bandura \& Schunk, 1981).

Nesse sentido, em muitas de suas obras, Bandura $(1986,1993,1997)$ demonstra que os julgamentos de autoeficácia funcionam como mediadores entre as reais capacidades do sujeito e o seu desempenho. A baixa autoeficácia percebida pode restringir a escolha de trabalho e influenciar a execução e persistência em alcançar a profissão escolhida. Por outro lado, a alta autoeficácia resulta em uma maior motivação para a realização das escolhas e para a busca de níveis mais altos, incluindo as escolhas de carreira, como posteriormente confirmado por outros estudiosos dessa vertente teórica (Lent, 2004; Pajares, 2002; Vega, Contreras, Chávez, \& Delgado, 2012).

\section{Autoeficácia no meio acadêmico}

Universitários que possuem crenças de autoeficácia positivas podem comprometer-se com seu futuro

2 profissional de maneira mais ativa, por meio de condutas vocacionais exploratórias, durante o período da 
graduação (Bardagi \& Hutz, 2012). Essas condutas referem-se a ações constantes na preparação do indivíduo para escolha de seu futuro profissional. Alguns autores referem-se à existência de quatro momentos marcantes na carreira do jovem. O primeiro está cercado por sentimentos de entusiasmo pela aprovação no vestibular. Em seguida, há um momento marcado por decepções relativas à instituição de ensino e aos professores, bem como preocupações acerca da escolha profissional (Bardagi, Lassance, \& Paradiso, 2003; Teixeira et al., 2012). Mais adiante, existe um interesse pela continuidade do curso e pelas atividades nas quais se está envolvido, o que reforça a satisfação com a escolha feita. Por fim, com o aumento das atividades do curso, há um envolvimento maior que auxilia na decisão de carreira e na autoeficácia vocacional, além de reforçar as expectativas relacionadas à inserção no mercado de trabalho (Sparta, Bardagi, \& Andrade, 2005; Teixeira, 2008).

Uma das formas de acompanhamento do estudante ao longo de sua formação ocorre com o uso de instrumentos de avaliação, cujos dados obtidos permitam informações válidas e fidedignas sobre o fenômeno em apreço (Urbina, 2007). Com relação à medida de autoeficácia, Teixeira (2008) realizou um estudo com a versão portuguesa, desenvolvida por Bandura (1990), da Escala Multidimensional de Autoeficácia Percebida (MSPSE, Multidimensional Scales of Perceived Self Efficacy), verificando que a escala era apropriada para medir aquilo a que se propunha.

Com base na MSPSE, Polydoro e Guerreiro-Casanova (2010) construíram a escala que foi usada neste estudo, a qual avalia as crenças de estudantes em sua capacidade frente a tarefas relacionadas à sua formação. Como existem especificidades e exigências inerentes aos diferentes níveis de ensino, para o domínio da experiência no ensino superior, as autoras propõem o uso do termo autoeficácia na formação superior. Assim, elas definem esse construto como o conjunto de crenças do estudante em sua capacidade para planejar e executar as ações necessárias para cumprir as exigências de tarefas acadêmicas específicas, quais sejam: motivação, autoconceito, objetivos acadêmicos e comprometimento institucional.

Salienta-se que as crenças de autoeficácia são importantes no processo de motivação, influenciando a forma como o sujeito se prepara para a ação. Indivíduos com elevados níveis de autoeficácia preferem desenvolver tarefas mais desafiadoras e definem para si próprios objetivos mais exigentes. No caso de universitários, seriam aqueles que investem mais nas tarefas e empreendem maiores níveis de esforço e persistência, ultrapassando mais rapidamente as dificuldades com que se confrontam e mantendo o foco nos objetivos definidos (Araújo et al., 2016; Costa, Araújo, \& Almeida, 2014).

Dois estudos que avaliaram diferença de autoeficácia acadêmica entre homens e mulheres apresentaram resultados diferentes. A respeito, Vega et al. (2012) verificaram que mulheres apresentaram escores mais elevados de autoeficácia acadêmica, o que não havia sido detectado em estudo anterior de Sparta e Gomes (2005). A existência dessa incongruência nos resultados incita a exploração de possíveis diferenças relacionadas à variável sexo, como a aqui realizada.

\section{Satisfação acadêmica}

Assim como a autoeficácia, a satisfação acadêmica também tem sido estudada com o objetivo de compreender o processo de vivências acadêmicas que ocorrem ao longo da graduação dos indivíduos (Bardagi \& Boff, 2010; Bardagi \& Hutz, 2010). A esse respeito, Santos, Polydoro, Scortegagna e Linden (2013) afirmam que as vivências dos alunos no ensino superior ao longo dos anos de curso são importantes, pois ocupam um papel relevante na busca por melhores condições de vida e de trabalho, que muitos almejam com a formação profissional. As autoras ainda esclarecem que a satisfação acadêmica se mostra relevante para compreensão da experiência acadêmica dos alunos, visto que elas identificaram diferenças entre os cursos pesquisados em uma mesma instituição, o que justifica a exploração de possíveis diferenças entre os cursos aqui focalizados. 
A satisfação acadêmica pode ser definida como uma variável cognitivo-afetiva que reflete a avaliação subjetiva dos alunos, relacionada à qualidade da sua experiência acadêmica (Almeida, Guisande, Soares, \& Saavedra, 2006; Soares \& Almeida, 2011). Embora o futuro do sujeito não esteja exclusivamente atrelado à sua opção profissional, as questões vocacionais tornam-se cada vez mais importantes, constituindo assim um campo de pesquisa da psicologia (Bardagi et al., 2003).

Alguns estudos que investigam a satisfação de vida entre universitários, optam por pesquisar sujeitos de uma área ou curso específico, tal como abordado por Santos et al., 2013. Por sua vez, Bardagi et al. (2003) afirmam que a principal fonte de satisfação está relacionada com a identificação pessoal com o curso escolhido, mostrando que estar comprometido com a escolha feita promove um maior bem-estar psicológico, além de potencializar uma avaliação otimista das possibilidades nas escolhas de resultados e minimizar as eventuais dificuldades. Estudos sobre a adaptação ao ensino superior têm apontado para a existência dessas relações (Araújo et al., 2014; Costa et al., 2014).

Pensando no contexto da formação superior, a hipótese levantada é de que os estudantes que apresentam crenças mais elevadas na própria capacidade de realizar com sucesso o que lhes é proposto, revelada pela autoeficácia percebida, mostram satisfação com o curso escolhido, uma vez que pessoas felizes com a atividade que exercem estão mais satisfeitas com a vida e, por extensão, com a experiência acadêmica. Assim, pode-se pensar que o aluno que usufrui daquilo que lhe é ofertado tanto pela instituição quanto pelos professores, sente-se mais engajado com o ambiente universitário, o que pode refletir as suas crenças ou capacidades em realizar as tarefas propostas.

A associação entre a autoeficácia para ensinar e a satisfação no trabalho foi investigada por lochite, Azzi, Polydoro e Winterstein (2011) com professores de Educação Física. Os autores constataram que os professores mais satisfeitos foram aqueles que se sentiam mais autoeficazes para a realização da tarefa de ensinar. Em termos teóricos, afirmam que, sob a perspectiva da teoria sociocognitiva, a autoeficácia docente e a satisfação com o trabalho, bem como a disposição em continuar ensinando, podem ser vistas como determinantes recíprocos. Nesse sentido, a pergunta que se pretende responder neste artigo é se o mesmo fenômeno ocorre com estudantes universitários.

Estudo anterior de Bardagi e Hutz (2010) demonstra haver relação direta do envolvimento do aluno com a instituição na qual está inserido, como parte de sua trajetória educacional. A forma como o aluno aproveita as oportunidades oferecidas e a estrutura física da instituição, assim como utiliza o acervo da biblioteca, e seu compromisso com o aprendizado, vai descrever a sua integração ao ensino superior (Santos et al., 2013).

A síntese dos estudos recuperados mostra que tanto as crenças de autoeficácia como a satisfação acadêmica são favoráveis à emissão de comportamentos de integração e adaptação aos novos padrões de atitudes exigidas no ensino superior, incluindo as exploratórias de carreira. Sob essa perspectiva, este estudo objetiva avaliar o possível papel preditivo da autoeficácia, no contexto acadêmico, em relação à satisfação com a experiência acadêmica. Ademais, buscam-se avaliar, exploratoriamente, possíveis diferenças entre universitários do sexo masculino e do feminino e, também, entre alunos de diferentes cursos, na percepção da autoeficácia e satisfação.

\section{Método}

\section{Participantes}

Participaram da pesquisa 372 alunos de uma instituição de ensino superior paulista, com idade entre 417 e 53 anos $(M=22,84 ; S D=6,09)$, sendo $66,4 \%$ do sexo feminino. Os universitários foram provenientes 
dos cursos de Psicologia, Arquitetura e Urbanismo, Administração, Engenharia Civil, Engenharia Química, Engenharia Mecânica, Engenharia da Produção, Engenharia Elétrica e Engenharia da Computação. Mesmo sendo uma amostra de conveniência, foram definidos como critérios de inclusão a idade mínima de 17 anos e a matrícula nas disciplinas oferecidas na ocasião da coleta de dados. Após explicação do propósito da pesquisa para as coordenações dos cursos de graduação, foram incluídos os cursos cujos coordenadores julgaram relevante obter informações referentes à autoeficácia e à satisfação acadêmica de seus alunos, que são os construtos aqui focalizados.

\section{Instrumentos}

Escala de Autoeficácia na Formação Superior (Polydoro \& Guerreiro-Casanova, 2010)

Esse instrumento avalia as crenças de estudantes em sua capacidade frente às tarefas da formação superior. Ele possui 34 itens dispostos em formato Likert, cuja atribuição de pontos varia de 1 (pouco capaz) a 10 (muito capaz). Evidências de validade do instrumento foram baseadas na análise da estrutura interna dos itens, que indicou cinco fatores coerentes teoricamente e que explicaram aproximadamente $56,9 \%$ da variância. Os fatores obtidos na análise fatorial foram: (1) Autoeficácia acadêmica, que avalia a percepção dos estudantes sobre a confiança em sua capacidade de aprender, demonstrar e aplicar o conteúdo do curso; (2) Autoeficácia na regulação da formação, referente à confiança percebida na capacidade de estabelecer metas, fazer escolhas, planejar e autorregular suas ações no processo de formação e desenvolvimento de carreira; (3) Autoeficácia em ações pró-ativas, que diz respeito à identificação da confiança percebida na capacidade de aproveitar as oportunidades de formação, atualizar os conhecimentos e promover melhorias institucionais; (4) Autoeficácia na interação social, que avalia a percepção dos estudantes sobre a confiança em sua capacidade de se relacionar com os colegas e professores, com fins acadêmicos e sociais; (5) Autoeficácia na gestão acadêmica, que se refere à confiança percebida na capacidade de se envolver, planejar e cumprir prazos em relação às atividades acadêmicas. A estimativa de precisão total do instrumento foi realizada com a análise de sua consistência interna, que foi considerada muito satisfatória, visto que o resultado obtido foi de $\alpha=0,95$. Considerando-se o alfa de cada fator houve variação de 0,80 a 0,88.

Escala de Satisfação com a Experiência Acadêmica (Schleich, Polydoro, \& Santos, 2006)

Essa escala mede o grau de satisfação atribuído pelo sujeito a diferentes aspectos da sua experiência acadêmica. A escala tem 34 itens dispostos em escalas Likert, cujas alternativas variavam de 1 (nada satisfeito) a 5 (totalmente satisfeito). Como principal evidência de validade, a escala foi submetida à análise da estrutura interna dos itens por meio da análise fatorial, que apontou uma estrutura de três fatores: (1) Satisfação com o curso, referente ao relacionamento com professores e colegas do curso, relevância de conteúdos e disciplinas; (2) Oportunidade de desenvolvimento, que abrange os eventos sociais oferecidos pela instituição, o currículo do curso e o envolvimento pessoal nas atividades do curso; e (3) Satisfação com a instituição, que diz respeito ao acervo disponível na biblioteca, recursos e equipamentos audiovisuais disponíveis e infraestrutura física da instituição. Em termos de estimativa de precisão, foi obtido o índice de consistência interna, cujo alfa geral da escala foi de 0,94. Considerando-se o alfa de cada fator, houve variação de 0,87 a 0,90.

\section{Procedimentos}

A pesquisa recebeu aprovação do Comitê de Ética em Pesquisa da Universidade São Francisco, sob o $n^{\circ}$ 29170214.5.0000.5514. Os objetivos da pesquisa foram expostos juntamente com o Termo de 
Consentimento Livre e Esclarecido (TCLE) para todos os participantes. Após concordarem com a participação, os alunos responderam coletivamente a ambos os instrumentos em sala de aula. O tempo de resposta aos instrumentos foi de, aproximadamente, trinta minutos.

Com o intuito de avaliar, exploratoriamente, possíveis diferenças de autoeficácia e satisfação com o curso, entre homens e mulheres e entre os cursos pesquisados (variáveis independentes), realizaram-se testes " $t$ " e ANOVA one-way, tendo como variáveis dependentes as dimensões de autoeficácia e satisfação com o curso. Não foi identificada nenhuma diferença estatisticamente significativa de autoeficácia e satisfação com o curso entre sexo e grupos $(p<0,05)$. Para avaliar o quanto as facetas da autoeficácia predizem a variância da satisfação acadêmica, foi realizada uma regressão múltipla (método Enter) tendo como variáveis preditoras as facetas da autoeficácia e, como variável critério, a satisfação acadêmica. O método de regressão Enter computa coeficientes de regressão das variáveis preditivas em relação à variável critério (e.g., satisfação acadêmica) e os apresenta em uma equação de regressão. Os pesos dos coeficientes indicam a relevância de cada variável para a predição. Ademais, é possível avaliar o total de variância final predito da variável critério, a partir do conjunto de variáveis preditoras.

\section{Resultados}

O modelo de regressão múltipla investigado (Tabela 1) indicou que o conjunto de facetas da autoeficácia acadêmica explicou grande parte da variância da satisfação com a experiência acadêmica total (aproximadamente 64\%), o que indica a relevância da autoeficácia para a percepção de satisfação no contexto acadêmico. Contudo, nem todas as facetas da autoeficácia apresentam a mesma relevância preditiva, sendo que apenas a autoeficácia na interação social apresentou coeficiente de regressão significativo $(\beta=0,79 ; p<0,001)$. Esse resultado não deve ser interpretado, entretanto, como se as demais dimensões da autoeficácia não fossem relevantes para a satisfação acadêmica, já que suas correlações com as demais facetas estão acima de 0,52. Essa aparente contradição ocorre porque no modelo de regressão é utilizada, para predição da satisfação acadêmica, apenas a parcela de variância exclusiva de cada faceta da autoeficácia. Como há considerável correlação das próprias facetas (valores acima de 0,50) e da variância da autoeficácia com a interação social, apenas esta última ganhou destaque no modelo. Em suma, esses resultados indicam que grande parte da variância da satisfação acadêmica pode ser predita, exclusivamente, pela autoeficácia com a interação social $(r=0,80)$, embora as demais facetas também possam contribuir para satisfação acadêmica.

Tabela 1

Modelo de regressão múltipla para investigar o papel preditivo da autoeficácia em relação à satisfação acadêmica

\begin{tabular}{lcccc}
\hline Preditores & $\beta$ & $t$ & $p$ & $R^{2}$ \\
\hline AE Acadêmica & $-0,026$ & $-0,628$ & 0,530 \\
AE Regulação da Formação & $-0,030$ & $-0,431$ & 0,667 \\
AE Ações Pró-Ativas & $-0,017$ & $-0,300$ & 0,765 \\
AE Interação Social & 0,792 & 6,395 & 0,000 \\
AE Gestão Acadêmica & 0,070 & 0,739 & 0,460 & 0,64 \\
& & & & 0 \\
\hline
\end{tabular}

Nota: AE: Autoeficácia; $\beta$ (Beta): Coeficiente de regressão padronizado; $R^{2}$ : Proporção total de variância explicada pelo modelo. 


\section{Discussão}

Este manuscrito buscou avaliar o possível papel preditivo da autoeficácia na formação superior em relação à satisfação com a experiência acadêmica. Como verificado pelo modelo de regressão, a satisfação com a experiência acadêmica é, consideravelmente, predita pela autoeficácia na formação superior. A autoeficácia com a interação social parece ser a faceta mais relevante para a satisfação com a experiência acadêmica. Esses resultados indicam que os alunos mais autoeficazes socialmente tendem a se mostrar mais satisfeitos com o curso escolhido, mas também que a satisfação com o curso pode depender, em grande parte, da capacidade de interação dos alunos.

É importante salientar que os achados deste estudo são congruentes com os pressupostos teóricos da teoria sociocognitiva com base em Bandura $(1977,1986,1993,1997)$. Com base nos mesmos pressupostos, Polydoro e Guerreiro-Casanova (2010) verificaram que alunos que relataram experiências insatisfatórias no ensino superior também apresentaram baixos níveis de autoeficácia nas relações sociais com colegas e professores - o que foi evidenciado por falta de feedback e de relações adequadas.

É possível que o desenvolvimento de habilidades como ser solidário e cooperativo com colegas, ter amigos para compartilhar dificuldades, pedir material emprestado, estudar e apresentar trabalhos em grupo podem ser comportamentos essenciais para o desenvolvimento da satisfação acadêmica. Deve-se ressaltar, contudo, que a autoeficácia em relação a outros domínios investigados também pode contribuir para a satisfação com o ensino superior, e esses achados merecem nota.

Autoeficácia acadêmica, autoeficácia na regulação da formação, autoeficácia em ações pró-ativas e autoeficácia na gestão acadêmica constituem habilidades desejáveis que favorecem a adaptação acadêmica geral. Dessa forma, estudantes mais aptos a se envolverem em projetos, cumprirem prazos, dedicarem-se aos estudos podem experienciar vivências mais positivas e satisfatórias, o que pode auxiliá-los a superar dificuldades ao ingressarem no ensino superior (Araújo et al., 2016). Os resultados obtidos no presente estudo são congruentes com a direção teórica e empírica encontrada em muitos outros anteriormente realizados (Ambiel, 2015; Bardagi \& Hutz, 2012; lochite et al., 2011; Magalhães \& Teixeira, 2013; Santos et al., 2013). Além disso, indicam que o desenvolvimento dessas habilidades pode ser crítico para o sucesso e satisfação acadêmica e poderia ser alvo de intervenções conduzidas por técnicos, professores, diretores, gestores ou outros.

A importância da autoeficácia em universitários não se restringe à satisfação com o curso. Em um estudo que objetivou verificar a relação entre as crenças de autoeficácia e o engagement (estado afetivo-cognitivo positivo, caracterizado por elevados níveis de energia e resistência mental, entre outros), Costa et al. (2014) constataram forte associação entre essas variáveis - o que indica que o desenvolvimento da autoeficácia pode favorecer o comprometimento e investimento dos alunos nas aulas e atividades propostas. Os achados do presente estudo são congruentes com esses resultados.

\section{Conclusão}

Em síntese, pode-se afirmar que os resultados suportam a hipótese do presente estudo, que apontou para a autoeficácia como preditora da satisfação com a experiência acadêmica em universitários. Esse resultado sugere que as instituições de ensino superior poderiam obter ganhos ao promover possibilidades de interação entre seus alunos (Almeida et al., 2006) dentro e fora da sala de aula. Intervenções desse tipo poderiam produzir ganhos para os alunos, que se sentiriam menos incapazes, isolados e frustrados, e também para os professores, que poderiam ter alunos mais satisfeitos e engajados. 
Este estudo não é livre de limitações. A amostra desta pesquisa é composta por estudantes de uma única cidade e do período noturno. Ou seja, esta amostra é pouco representativa da população de universitários brasileiros, o que não permite a generalização dos achados. Ademais, devido ao delineamento correlacional usado nesta investigação, não se pode inferir causalidade entre autoeficácia e satisfação acadêmica.

Com o intuito de avançar na compreensão sobre a relação dessas variáveis, sugere-se que novos estudos tentem identificar novas variáveis e explicar os mecanismos existentes entre as relações da autoeficácia e satisfação acadêmica. Sugere-se que tais investigações sejam conduzidas em amostras mais representativas do universo acadêmico. O descobrimento de fatores que impactam na satisfação acadêmica pode subsidiar intervenções mais específicas e contribuir para o sucesso e satisfação de muitos universitários em um período crítico do desenvolvimento.

\section{Colaboradores}

A.A.A. SANTOS participou da concepção geral da pesquisa, redação, análise de dados e revisão geral. C. ZANON realizou a redação, análise de dados e revisão geral. V.D. ILHA foi responsável pela concepção geral da pesquisa, redação e revisão geral.

\section{Referências}

Almeida, L. S., Guisande, M. A., Soares, A. P., \& Saavedra, L. (2006). Acesso e sucesso do ensino superior em Portugal: questões de género, origem sócio-cultural e percurso académico dos alunos. Psicologia: Reflexão e Crítica, 19(3), 507-514. http://dx.doi.org/10.1590/S0102-79722006000300020

Ambiel, R. A. M. (2015). Construção de escalas de motivos para evasão do ensino superior. Avaliação Psicológica, 14(1), 41-52. http://dx.doi.org/10.15689/ap.2015.1401.05

Araújo, A. M., Almeida, L. S., Ferreira, J. C., Santos, A. A. A., Noronha, A. P. P., \& Zanon, C. (2014). Questionário de Adaptação ao Ensino Superior (QAES): construção e validação de um novo questionário. Psicologia, Educação e Cultura, 18(1), 131-145. Recuperado em janeiro 10, 2016, de http://www.cic.pt/pec/default.asp?link=N28

Araújo, A. M., Santos, A. A. A., Noronha, A. P. P., Zanon, C., Ferreira, J. A., \& Almeida, L. S. (2016). Dificuldades antecipadas de adaptação ao ensino superior: um estudo com estudantes do primeiro ano. Revista de Estudios e Investigación en Psicología y Educación, 3(2), 102-111. http://dx.doi.org/10.17979/reipe.2016.3.2.1846

Bandura, A. (1977). Self-Eficacy: Toward a unifying theory of behavioral change. Psychological Review, 84(2), 191-215. Retrieved January 10, 2016, from https://www.uky.edu/ eushe2/Bandura/Bandura1977PR.pdf

Bandura, A. (1986). From thought to action: Mechanisms of personal agency. The New Zealand Journal of Psychology, 15(1), 1-17. Retrieved January 10, 2016, from http://www.psychology.org.nz/wp-content/uploads/NZJP-Vol151-19861-Bandura.pdf

Bandura, A. (1990). Multidimensional scales of perceived academic efficacy. Stanford: Stanford University.

Bandura, A. (1993). Perceived self-efficacy in cognitive development and functioning. Educational Psychologist, 28(2), 117-148. Retrieved January 10, 2016, from https://www.uky.edu/ eushe2/Bandura/Bandura1993EP.pdf

Bandura, A. (1997). Self-efficacy: The exercise of control. New York: Freeman.

Bandura, A., \& Schunk, D. H. (1981). Cultivating competence, self-efficacy, and intrinsic interest through proximal self-motivation. Journal of Personality and Social Psychology, 41(3), 386-589. Retrieved January 10, 2016, from https:// www.uky.edu/ eushe2/Bandura/Bandura1981JPSP.pdf

Bardagi, M. P., \& Boff, R. M. (2010). Autoconceito, auto-eficácia profissional e comportamento exploratório em universitários concluintes. Avaliação, 15(1), 41-56. http://dx.doi.org/10.1590/S1414-40772010000100003

Bardagi, M. P., \& Hutz, C. S. (2010). Satisfação de vida, comprometimento com a carreira e exploração vocacional em estudantes universitários. Arquivos Brasileiros de Psicologia, 62(1), 159-170. Recuperado em janeiro 10, 2016, de http://pepsic.bvsalud.org/scielo.php?script=sci_arttext\&pid=S1809-52672010000100016

Bardagi, M. P., \& Hutz, C. S. (2012). Rotina acadêmica e relação com colegas e professores: impacto na evasão universitária. Psico, 43(2), 174-184. Recuperado em janeiro 10, 2016, de http://pepsic.bvsalud.org/pdf/rbop/v14n2/07.pdf 
Bardagi, M. P., Lassance, M. C. P., \& Paradiso, A. C. (2003). Trajetória acadêmica e satisfação com a escolha profissional de universitários em meio de curso. Revista Brasileira de Orientação Profissional, 4(1/2), 153-166. Recuperado em janeiro 10, 2016, de http://pepsic.bvsalud.org/scielo.php?script=sci_arttext\&pid=S1679-33902003000100013

Carlotto, R. C., Teixeira, M. A. P., \& Dias, A. C. G. (2015). Adaptação acadêmcia e coping em estudantes universitários. Psico-USF, 20(3), 421-432. http://dx.doi.org/10.1590/1413-82712015200305

Costa, A. R., Araújo, A. M., \& Almeida, L. S. (2014). Relação entre a percepção da autoeficácia acadêmica e o engagement de estudantes de Engenharia. International Journal of Developmental and Educational Psychology, 2(1), 307-314. Recuperado em janeiro 10, 2016, de https://repositorium.sdum.uminho.pt/bitstream/1822/32004/1/Relação\%20 entre \%20a\%20percepção\%20da\%20autoeficácia \%20académica\%20e\%200\%20engagement\%20de\%20 estudantes\%20de\%20engenharia.pdf

lochite, R. T., Azzi, R. G., Polydoro, S. A. J., \& Winterstein, P. J. (2011). Autoeficácia docente, satisfação e disposição para continuar na docência por professores de educação física. Revista Brasileira de Ciências do Esporte, 33(4), 825-839. http://dx.doi.org/10.1590/S0101-32892011000400003

Lent, R. (2004). A social cognitive view of career development and counseling. In S. D. Brown \& R. W. Lent (Eds.), Career development and counseling: Putting theory and research to work (pp.101-130). Hoboken: John Wiley and Sons.

Magalhães, M. O., \& Teixeira, M. A. P. (2013). Antecedentes de comportamentos de busca de emprego na transição da universidade para o mercado de trabalho. Psicologia: Teoria e Pesquisa, 29(4), 411-419. http://dx.doi.org/10.1590/ S0102-37722013000400007

Ourique, L. R., \& Teixeira, M. A. P. (2012). Autoeficácia e personalidade no planejamento de carreira de universitários. Psico-USF, 17(2), 311-321. http://dx.doi.org/10.1590/S1413-82712012000200015

Pajares, F. (2002). Overview of social cognitive theory and of self-efficacy. Retrieved February 16, 2016, from http://www. emory.edu/EDUCATION/mfp/eff.html

Polydoro, S. A. J., \& Guerreiro-Casanova, D. C. (2010). Escala de autoeficácia na formação superior: construção e estudo de validação. Avaliação Psicológica, 9(2), 267-278. Recuperado em janeiro 10, 2016, de http://www.redalyc.org/ pdf/3350/335027283011.pdf

Santos, A. A. A., Polydoro, S. A. J., Scortegagna, S. A., \& Linden, M. S. S. (2013). Integração ao ensino superior e satisfação acadêmica em universitários. Psicologia: Ciência e Profissão, 33(4), 780-793. http://dx.doi.org/10.1590/ S1414-98932011000100006

Schleich, A. L. R., Polydoro, S. A. J., \& Santos, A. A. A. (2006). Escala de satisfação com a experiência acadêmica de estudantes do ensino superior. Avaliação Psicológica, 5(1), 11-20. Recuperado em setembro 5, 2016, de http://pepsic. bvsalud.org/pdf/avp/v5n1a03.pdf

Soares, A. P., \& Almeida, L. S. (2011). Questionário de satisfação académica. In C. Machado, M. Gonçalves, L. Almeida, \& M. R. Simões (Eds.), Instrumentos e contextos de avaliação psicológica (pp. 103-124). Coimbra: Almedina.

Sparta, M., Bardagi, M. P., Andrade, A. M. J. (2005). Exploração vocacional e exploração profissional percebida em estudantes carentes. Aletheia, 22(1), 79-88. Recuperado em janeiro 10, 2016, de http://www.redalyc.org/ pdf/1150/115013470008.pdf

Sparta, M., \& Gomes, W. (2005). Importância atribuída ao ingresso na educação superior por alunos do ensino médio. Revista Brasileira de Orientação Profissional, 6(2), 45-53. Recuperado em janeiro 10, 2016, de http://pepsic.bvsalud. org/pdf/rbop/v6n2/v6n2a05.pdf

Teixeira, M. O. (2008). A escala multidimensional de auto-eficácia percebida: um estudo exploratório numa amostra de estudantes de ensino superior. RIDEP, 25(1), 141-157. Recuperado em janeiro 10, 2016, de http://www.aidep. org/03_ridep/R25/R257.pdf

Teixeira, M. A. P, Castro, A. K. S. S., \& Zoltowski, A. P. C. (2012). Integração acadêmica e integração social nas primeiras semanas na universidade: percepções de estudantes universitários. Revista Interinstitucional de Psicologia, 5(1), $69-85$. Recuperado em janeiro 10, 2016, de http://pepsic.bvsalud.org/pdf/gerais/v5n1/v5n1a06.pdf

Urbina, S. (2007). Fundamentos da testagem psicológica. Porto Alegre: Artmed.

Vega, H. B., Contreras, M. O., Chávez, J. F. A., \& Delgado, J. C. G. (2012). Autoeficácia percebida en conductas acádemicas, diferencias entre hombres y mujeres. Revista Mexicana de Investigación Educativa, 17(53), 557-571. Recuperado el Enero 10, 2016, del http://www.scielo.org.mx/pdf/rmie/v17n53/v17n53a11.pdf

Recebido: maio 10, 2016

Versão final: fevereiro 13, 2017

Aprovado: fevereiro 16, 2017 